\title{
Dual Transition Uniform Lbp Matrix for Efficient Image Retrieval
}

\author{
V.Vijaya Kumar \\ Anurag Group of Institutions, Hyderabad, India \\ Email: vakula_vijay@yahoo.com \\ A. Srinivasa Rao \\ Research Scholar, Krishna University, Machilipatnam, India \\ Email: akella.srinivas08@gmail.com \\ Prof.YK Sundara Krishna \\ Professor in CSE Dept. Krishna University, Machilipatnam, India \\ Email: yksk2010@gmail.com
}

\begin{abstract}
Texture image retrieval plays a significant and important role in these days, especially in the era of bigdata. The big-data is mainly represented by unstructured data like images, videos and messages etc. Efficient methods of image retrieval that reduces the complexity of the existing methods is need for the big-data era. The present paper proposes a new method of texture retrieval based on local binary pattern (LBP) approach. One of the main disadvantages of LBP is, it generates 256 different patterns on a $3 \times 3$ neighborhood and a method based on this for retrieval needs 256 comparisons which is very tedious and complex. The retrieval methods based on uniform LBP's which consists of 59 different patterns of LBP is also complex in nature. To overcome this, the present paper divided LBP into dual LBP's consisting four pixels. The present paper based on this dual LBP derived a 2-dimensional dual uniform LBP matrix (DULBPM) that contains only four entries. The texture image retrieval is performed using these four entries of DULBPM. The proposed method is evaluated on the animal fur, car, leaf and rubber textures.
\end{abstract}

Index Terms-Dimensionality, Big-data, Complexity, Local binary pattern, Dual uniform local binary matrix.

\section{INTRODUCTION}

There are many general-purpose image retrieval systems are proposed in the literature [1]. One of the popular among these retrieval systems is content-based image retrieval (CBIR). CBIR critically and accurately assist users in finding visually related images with in huge collection of images.In a typical CBIR approach, a user submits an image based query and that can be used by the application to extract visual features from image. The visual features may include by a set of quantitative features, extracted from regions of interest (ROIs) of the image like shape, color, texture and local features depending upon the type of image retrieval system being used. These features are used in order to search and retrieve similar images from image database. The CBIR system finds an appropriate distance between the query image and feature data set to find the best matches in the corresponding feature space [2]. By this the image retrieval system displays images which are having closest similarity that can be used by application. Therefore the main motive behind CBIR is, to search for similar images directly on their visual content. In addition, the performance of CBIR systems is often constrained by the low-level properties of these features because they cannot effectively model the user's high-level expectations [3]. This is referred in the literature as the "semantic gap" problem.

Plenty of literature is available showing problems related to text-based query, for example, search experience and domain expertise can affect the content based image retrieval (CBIR) performance $[4,5]$. In the similar way an ill-defined problems or queries can produce poor results. The previous works on CBIR are suffering with many contextual factors like interaction time [6], user's subjective perception of relevance [7, 8] and environmental settings [9, 10]. Even today many researchers are working on approaches based on CBIR and it still under investigation. The knowledge of how relevant the particular piece of information (document or image) is to the user and how its content can be reused in order to find documents or images that are similar [11]. Documents or images that are similar to the relevant content have a very high probability of relevance [12]. To address the various problems of image retrieval based on CBIR various researchers proposed a variety of algorithms. Most of these approaches ignored the existence of others [13-19]. Methods based on one specific algorithm (e.g., color, texture or shape) can work effectively only on specific types of images. Local binary pattern (LBP) [27] is very useful and popular approaches recently found for CBIR, because it describes the local contents and attributes significantly which is a very useful tool. The features derived based on LBP are used 
by many researchers in wide spread of applications like texture classification, face recognition, facial expression recognition, age classification, medical image processing etc. [20-26]. The present paper reduces overall dimensionality of LBP and thus reduced the complexity and derived dual transition uniform LBP matrix (DULBPM) for efficient image retrieval.

The rest of the paper is organized as follows. Section II delivers about related work and section III delivers the proposed approach. Section IV presents results achieved by applying the proposed approach on different texture images. Section V concludes the paper.

\section{RELATED WORK}

The proposed Dual matrix is derived from the basic LBP operators. The Local Binary Pattern (LBP) was introduced by Ojala et al [27] in 1996. LBP is simple, computationally efficient, robust, and derives local attributes efficiently. With these features, many researchers started working with LBP in various domains and especially in face recognition [1, 31, 32, 39]. The LBP is a powerful tool to describe the local attributes of a texture. In the LBP the grey level image is converted into binary by taking the central pixel value as a threshold and this grey level value is compared with its neighborhood values. The resulting binary valued image is treated as a local descriptor. The basic LBP was initially derived on a 3*3 neighborhood. This LBP operator can also be represented with different variation of $(P, R)$ where $P$ represents the number of neighborhood pixels and $R$ is the Radius. By this the basic LBP operator is represented as $(8,1)$. The 8 -bit binary representation or 8 -neighboring pixels on a $3 * 3$ neighborhood or $(8,1)$ derives a LBP code that ranges from 0 to 255 .

The LBP operator takes the following form as given in equation 1.

$$
\operatorname{LBP}_{(8,1)}=\sum_{n=0}^{7} 2^{n} S\left(P_{c}-P_{n}\right)
$$

Where ' $n$ ' runs over the 8 neighbors (0 to 7$)$ of the central pixel $\mathrm{C}, \mathrm{P} \_$cand $\mathrm{P} \_\mathrm{n}$ are the grey level intensities at $\mathrm{c}$ and $\mathrm{n}$ and $\mathrm{S}(\mathrm{u})$ will be 1 if $\mathrm{u} \quad 0$ and 0 otherwise. The LBP encoding process on a $3 * 3$ neighborhood i.e. $(8,1)$ is given in Fig. 1

\begin{tabular}{|c|c|c|}
\hline 16 & 21 & 6 \\
\hline 14 & 8 & 7 \\
\hline 9 & 7 & 5 \\
\hline
\end{tabular}

(a)

$$
\begin{gathered}
\mathrm{P}_{\mathrm{c}} \text { as } \\
\text { threshold } \\
\mathrm{P}_{\mathrm{c}}=8
\end{gathered}
$$

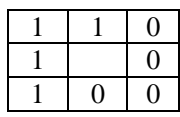

(b)
Binary Code: 11000011 LBP Code: 195 (c)
Fig. 1. Encoding of basic LBP operator.

The binary weights of LBP can be given in eight different ways as shown in Fig.2.

The value of the LBP changes by the representation of the weights. The LBP can be calculated in 8 different ways for a $3 \times 3$ neighborhood as shown in Fig. 2. That is for any $3 \times 3$ neighborhood one can generate eight LBP values. The LBP value for the Fig.1 (a) in all eight directions as represented in Fig.2 is given as 227, 242,

\begin{tabular}{|c|c|c|}
\hline $2^{7}$ & $2^{0}$ & $2^{1}$ \\
\hline $2^{6}$ & & $2^{2}$ \\
\hline $2^{5}$ & $2^{4}$ & $2^{3}$ \\
\hline \multicolumn{3}{|c|}{ (b) } \\
\hline $2^{5}$ & $2^{6}$ & $2^{7}$ \\
\hline $2^{4}$ & & $2^{0}$ \\
\hline $2^{3}$ & $2^{2}$ & $2^{1}$ \\
\hline \multicolumn{3}{|c|}{ (d) } \\
\hline $2^{3}$ & $2^{4}$ & $2^{5}$ \\
\hline $2^{2}$ & & $2^{6}$ \\
\hline $2^{1}$ & $2^{0}$ & $2^{7}$ \\
\hline \multicolumn{3}{|c|}{ (f) } \\
\hline $2^{1}$ & $2^{2}$ & $2^{3}$ \\
\hline $2^{0}$ & & $2^{4}$ \\
\hline $2^{7}$ & $2^{6}$ & $2^{5}$ \\
\hline
\end{tabular}
$121,188,94,47,151$, and 203 respectively.

\begin{tabular}{|c|c|c|}
\hline $2^{0}$ & $2^{1}$ & $2^{2}$ \\
\hline $2^{7}$ & & $2^{3}$ \\
\hline $2^{6}$ & $2^{5}$ & $2^{4}$ \\
\hline \multicolumn{3}{|c|}{$(\mathrm{a})$} \\
\hline $2^{6}$ & $2^{7}$ & $2^{0}$ \\
\hline $2^{5}$ & $2^{1}$ & $2^{2}$ \\
\hline $2^{4}$ & $2^{3}$ & $(\mathrm{c})$ \\
\hline \multicolumn{3}{|c|}{} \\
\hline $2^{4}$ & $2^{5}$ & $2^{6}$ \\
\hline $2^{3}$ & $2^{1}$ & $2^{0}$ \\
\hline $2^{2}$ & $(\mathrm{e})$ \\
\hline \multicolumn{2}{|c|}{} \\
\hline $2^{2}$ & $2^{3}$ & $2^{4}$ \\
\hline $2^{1}$ & $2^{7}$ & $2^{6}$ \\
\hline $2^{0}$ & $2^{7}$ & $(\mathrm{~g})$
\end{tabular}

Fig. 2. Eight Different Ways of measuring LBP weights on a $3 \times 3$ neighborhood.

Many researchers worked on Uniform Local Binary Pattern (ULBP) and Non Uniform Local Binary Pattern (NULBP) and derived many conclusions. An LBP is uniform if it contains at most one - zero to one and one one to zero transition in a circular manner. For example 11111111 (0 transitions), 00000001 (2 transitions) are uniform, whereas 11001100 (4 transitions), and 01011111 (5 transitions), 10101100 (6 transitions), 01010101 (8 transitions) are not uniform. Some of the researchers [1, 14, 19, 27, 28, 44] considered only ULBP's for classification, recognition and for solving other problems because of the following reasons. a) ULBP's are treated as the fundamental properties of texture image. b) 80 to $85 \%$ of the texture images contain only ULBP's. c) There are 192 NULBP's and treating them as miscellaneous will reduce lot of dimensionality without losing much of the texture content. The other group of researchers [29,30,31,32,33,34,35 ] considered a part or few of NULBP's along with ULBP's and proved that this combination yielded a better or a little progress than by considering only ULBP's. From this one can understand that ULBP's can be treated as the fundamental properties of the texture image, however considering them only may lose some basic information. Therefore it is better to consider a sub set of NULBP's.

From the above discussion it is evident that the major problem is how to select a subset from NULPBP's to improve the overall performance and to reduce overall dimensionality. For this the present paper divided the basic LBP operator with 8 neighboring pixels into two four bit LBP operators on a radius of 1 . The present paper considered all transitions i.e uniform and non- uniform on the dual LBP, then derived a dual LBP matrix and image retrieval is performed on this.

\section{DeRIVATION Of The Proposed DUAL UNIFORM LBP MATRIX (DULBPM)}

LBP on a $3 \times 3$ neighborhood generates 0 to 255 i.e. 256 different patterns. One can retrieve the texture image 
based on the 256 LBP in the following way.

1. Find the individual histogram of 256 LBP's on the texture, by convolving the local $3 \times 3$ neighborhood in an overlapped manner. Place them in the feature library.

2. For texture image retrieval find the individual histograms of 256 LBP's on the query texture image.

3. Apply Euclidean distance measure between the individual histograms of the 256 LBP's of query texture image with database texture images.

4. Pick up the nearest one i.e. the database texture that exhibits shortest Euclidean distance with the query texture image. If the query and the retrieved texture images are same then it represents a hit otherwise a miss.

The major disadvantage of this method is due to its high complexity and dimensionality. In this method one should evaluate the histogram of all individual 256 LBP's and Euclidean distance should be found in between the 256 features.

Further research on LBP invented uniform LBP (ULPB) [28]. The 59 ULPB's out of 256 LBP's are named as uniform LBP's and they contains at most two bit wise circular transitions form 0 to 1 or 1 to 0 . The Ojala et.al [27] proved that most of the textures contain more than $80 \%$ of the patterns as ULBP. That's why ULBP represents the fundamental unit of LBP. One can retrieve images based on the histogram of ULBP's. This also becomes a complex task because one should find Euclidean distance between 59 patterns of query and feature database textures.

The aim of the present paper is to reduce this complexity. For this, the proposed method divided the local 3x3 neighborhood into dual sets of four pixels each. The first set is called diagonal LBP contains the four pixels $d_{1}, d_{2}, d_{3}, d_{4}$ as shown in Fig. $3 a$ in red color. The second set is named as corner LBP contains the corner pixels $\mathrm{c}_{1}, \mathrm{c}_{2}, \mathrm{c}_{3}, \mathrm{c}_{4}$ as shown in Fig. $3 \mathrm{a}$ in green color. The four pixels of diagonal LBP and corner LBP forms 16 LBP's each.

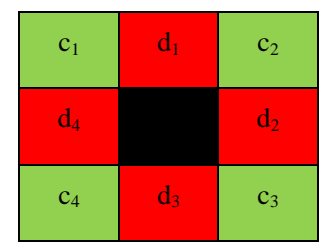

Fig. 3.a. Cross and diagonal pixel representation in LBP.

The four bit LBP forms zero, two and four transitions of 0 to 1 or 1 to 0 . The LBP codes $0(0000)$ and 15 (1111) forms zero transitions. The four bit circular LBP codes 1(0001), 2(0010), 3(0011), 4(0100), 6(0110), 7(0111), 8(1000), 9(1001), 11(1011), 12(1100), 13(1101) and 14(1110) forms 2 circular transitions from 0 to 1 or 1 to 0 . The four bit LBP codes 5(0101) and 10(1010), forms 4 circular transitions from 0 to 1 or 1 to 0 and they does not fall into ULBP group. The present paper evaluated and created a 2-D matrix that corresponds to the histogram of uniform transitions of DIAGONAL LBP versus corner LBP. The dual uniform LBP matrix (DULBPM) is a 2-D matrix and it is shown in Fig.3b.

\begin{tabular}{|c|c|c|}
\hline \multirow{2}{*}{$\begin{array}{c}\text { No. of uniform } \\
\text { transitions on } \\
\text { corner LBP }\end{array}$} & \multicolumn{2}{|c|}{$\begin{array}{c}\text { No. of uniform } \\
\text { transitions on } \\
\text { DIAGONAL LBP }\end{array}$} \\
\cline { 2 - 3 } & 0 & \\
\hline 0 & & \\
\hline 2 & & \\
\hline
\end{tabular}

Fig.3.b. The structure of DULBPM.

The rows of the proposed DULBPM contain the number of uniform transitions of corner LBP and columns contain the number of uniform transitions of diagonal LBP. The DULBPM $(i, j)$ refers to the frequency occurrence of the $i^{\text {th }}$ uniform transition of corner LBP versus of $j^{\text {th }}$ uniform transition of the diagonal LBP.

The Fig.4.a represents a LBP. The four pixels of corner LBP and diagonal LBP are represented with green and red color respectively. The corner LBP and diagonal LBP of Fig.4.a forms a four bit LBP codes of 1111 and 0110 respectively and they form 0 and 2 circular bitwise transitions from 0 to 1 or 1 to 0 respectively. This results the DULBPM entry as

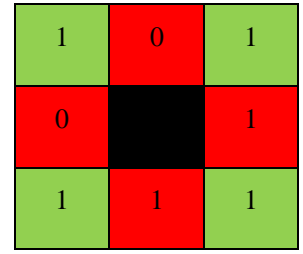

Fig. 4.a. LBP representation. corner $\mathrm{LBP}=1111=0$ transitions

diagonal $\mathrm{LBP}=0110=2$ transitions

$\operatorname{DULBPM}(0,2)=\operatorname{DULBPM}(0,2)+1$;

Fig.4.b. Representation of DULBPM.
$\operatorname{DULBPM}(0,2)=\operatorname{DULBPM}(0,2)+1$. This is shown in Fig. 4.b

In the similar way the four bit corner LBP and diagonal LBP of Fig.5.a exhibits 2 and 2 transitions respectively. This results the entry of DULBPM $(2,2)=$ DULBPM $(2$, 2) +1 .

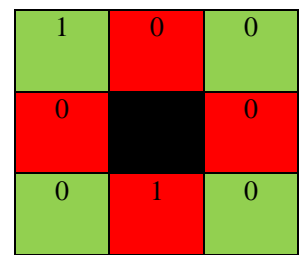

Fig. 5.a. LBP representation.
Corner $\mathrm{LBP}=1000=2$ transitions
Diagonal $\mathrm{LBP}=0010=2$ transitions

$\operatorname{DULBPM}(2,2)=\operatorname{DULBPM}(2,2)+1$;

Fig. 5.b. Representation of DULBPM.
The DULBPM dimensions are $2 \times 2$, means they contain only 4 entries. Initially the 2-D DULBPM is initialized to zero. The above process is repeated on the entire texture image to form DULBPM for each texture. The feature or training library database consists of DULBPM for each 
texture. The proposed DULBPM not considered the 4 transitions on corner LBP and diagonal LBP because they are not part of ULBP. If one considers them, then the proposed matrix becomes dual LBP matrix (DLBPM) and it contains 9 entries with a dimension of $3 \times 3$.

\section{RESULTS AND DisCUSSIONS}

To test the efficacy of the proposed DULBPM the present research tested this on animal fur, rubber, leaf and car texture images of resolution $256 \times 256$. These images are collected from a Google database. The DULBPM is a 2x2 2-D matrix i.e. it consists of 4 entries only and DULBPM entries are initialized to zero. DULBPM is evaluated for 1000 different textures of animal fur, rubber, leaf and car textures and placed in the feature or training database. Whenever a query texture image is given, the DULBPM is evaluated on this and the Euclidean distance is measured between the query texture image and all the textured images of the feature set. The texture of feature database that exhibits the minimum Euclidean distance is selected as a retrieved image. If the retrieved image exactly matches the query image, then it represent a hit otherwise a miss. The Table 1 represents the DULBPM entries of 20 animal fur texture images. In all the tables' col-1 and col-2 represents the 0 and 2 transitions of diagonal LBP and row- 1 and row- 2 represents the 0 and 2 transitions of corner LBP respectively. For example in Table 1 the DULBPM $(0,0)$ for A_F_1 texture represents a value 8042 . In the similar way in Table 1 for animal fur-4 texture (A_F_4) DULBPM $(2,2)=52661$. Table 2, 3 and 4 represents the 2-D DULBPM entries for rubber, leaf and car texture respectively. The texture retrieval is performed separately based on Euclidian distance between query texture image and feature database texture images using individual entries of DULBPM. That is based on 0 verses 0,0 verses 2,2 verses 0,2 verses 2 bitwise transition of corner LBP verses diagonal LBP.

The image retrieval rate is calculated using the $\operatorname{DULBPM}(0,2), \operatorname{DULBPM}(2,0)$ and $\operatorname{DULBPM}(2,2)$ entries for the above four group of textures. The Table 5 gives the texture image retrieval rate for different transition of DULBPM. From the table it is evident that the retrieval based on DULBPM $(2,0)$ and DULBPM $(0$, 2 ) entries shown higher performance. There are only two bitwise zero transitions on each of the diagonal and corner LBP, that's why the DULBPM $(0,0)$ attained a normal average retrieval rate of $53 \%$.
Table 1. DULBPM entries for animal fur texture.

\begin{tabular}{|c|c|c|c|}
\hline \multirow[t]{2}{*}{1} & \multirow{2}{*}{ A_F_1 } & 8042 & 3362 \\
\hline & & 3991 & 49121 \\
\hline \multirow[t]{2}{*}{2} & \multirow{2}{*}{ A_F_2 } & 8776 & 2910 \\
\hline & & 3320 & 49510 \\
\hline \multirow[t]{2}{*}{3} & \multirow{2}{*}{ A_F_3 } & 6003 & 3537 \\
\hline & & 3843 & 51133 \\
\hline \multirow[t]{2}{*}{4} & \multirow{2}{*}{ A_F_4 } & 6541 & 2098 \\
\hline & & 3216 & 52661 \\
\hline \multirow[t]{2}{*}{5} & \multirow{2}{*}{ A_F_5 } & 9335 & 3011 \\
\hline & & 3950 & 48220 \\
\hline \multirow[t]{2}{*}{6} & \multirow{2}{*}{ A_F_6 } & 9697 & 4169 \\
\hline & & 4073 & 46577 \\
\hline \multirow[t]{2}{*}{7} & \multirow{2}{*}{ A_F_7 } & 6314 & 2421 \\
\hline & & 3038 & 52743 \\
\hline \multirow[t]{2}{*}{8} & \multirow{2}{*}{ A_F_8 } & 13792 & 466 \\
\hline & & 915 & 49343 \\
\hline \multirow[t]{2}{*}{9} & \multirow{2}{*}{ A_F_9 } & 6499 & 5125 \\
\hline & & 5711 & 47181 \\
\hline \multirow[t]{2}{*}{10} & \multirow{2}{*}{ A_F_10 } & 8347 & 4342 \\
\hline & & 4430 & 47397 \\
\hline \multirow[t]{2}{*}{11} & \multirow{2}{*}{ A_F_11 } & 7229 & 5175 \\
\hline & & 5725 & 46387 \\
\hline \multirow[t]{2}{*}{12} & \multirow{2}{*}{ A_F_12 } & 8448 & 3929 \\
\hline & & 5165 & 46974 \\
\hline \multirow[t]{2}{*}{13} & \multirow{2}{*}{ A_F_13 } & 8448 & 3929 \\
\hline & & 5165 & 46974 \\
\hline \multirow[t]{2}{*}{14} & & 7230 & 5069 \\
\hline & & 6982 & 45235 \\
\hline 15 & & 9463 & 5181 \\
\hline & $A_{-} \Gamma_{-1}$ & 5669 & 44203 \\
\hline 16 & & 9270 & 2475 \\
\hline & A_5_10 & 3538 & 49233 \\
\hline 17 & & 6904 & 1942 \\
\hline & H_1_-1 & 3666 & 52004 \\
\hline 18 & 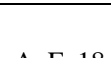 & 8731 & 2494 \\
\hline & $A_{-} F_{-} 18$ & 3926 & 49365 \\
\hline 19 & & 6220 & 4079 \\
\hline & & 6584 & 45635 \\
\hline 20 & & 8463 & 6281 \\
\hline & A_t_L & 7669 & 55203 \\
\hline
\end{tabular}


Table 2. DULBPM entries for rubber texture.

\begin{tabular}{|c|c|c|c|}
\hline \multirow[t]{2}{*}{1} & \multirow{2}{*}{ Rubber_1 } & 9527 & 1518 \\
\hline & & 2198 & 51273 \\
\hline \multirow[t]{2}{*}{2} & \multirow{2}{*}{ Rubber_2 } & 9349 & 2480 \\
\hline & & 3999 & 48688 \\
\hline \multirow[t]{2}{*}{3} & \multirow{2}{*}{ Rubber_3 } & 9673 & 1824 \\
\hline & & 2953 & 50066 \\
\hline \multirow[t]{2}{*}{4} & \multirow{2}{*}{ Rubber_4 } & 11172 & 2385 \\
\hline & & 2680 & 48279 \\
\hline \multirow[t]{2}{*}{5} & \multirow{2}{*}{ Rubber_5 } & 9103 & 2444 \\
\hline & & 3440 & 49529 \\
\hline \multirow[t]{2}{*}{6} & \multirow{2}{*}{ Rubber_6 } & 8442 & 2370 \\
\hline & & 3032 & 50672 \\
\hline \multirow[t]{2}{*}{7} & \multirow{2}{*}{ Rubber_7 } & 8855 & 3037 \\
\hline & & 2994 & 49630 \\
\hline \multirow[t]{2}{*}{8} & \multirow{2}{*}{ Rubber_8 } & 11408 & 2151 \\
\hline & & 2911 & 48046 \\
\hline \multirow[t]{2}{*}{9} & \multirow{2}{*}{ Rubber_9 } & 7992 & 3596 \\
\hline & & 4414 & 48514 \\
\hline \multirow[t]{2}{*}{10} & \multirow{2}{*}{ Rubber_10 } & 7460 & 3297 \\
\hline & & 3943 & 49816 \\
\hline \multirow[t]{2}{*}{11} & \multirow{2}{*}{ Rubber_11 } & 8827 & 1295 \\
\hline & & 3063 & 51331 \\
\hline \multirow[t]{2}{*}{12} & \multirow{2}{*}{ Rubber_12 } & 8519 & 1511 \\
\hline & & 2854 & 51632 \\
\hline \multirow[t]{2}{*}{13} & \multirow{2}{*}{ Rubber_13 } & 8833 & 2388 \\
\hline & & 3808 & 49487 \\
\hline \multirow[t]{2}{*}{14} & 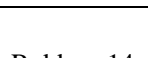 & 7980 & 2719 \\
\hline & & 4079 & 49738 \\
\hline 15 & & 12427 & 686 \\
\hline & Rubber_15 & 1500 & 49903 \\
\hline 16 & & 10991 & 1176 \\
\hline & Kubber_10 & 1839 & 50510 \\
\hline 17 & & 7090 & 2938 \\
\hline & Rubber_1/ & 4704 & 49784 \\
\hline 18 & & 12898 & 2345 \\
\hline & KubDer_18 & 7650 & 2675 \\
\hline 19 & & 13172 & 2885 \\
\hline & Kubber_19 & 1480 & 43229 \\
\hline 20 & D & 8153 & 2466 \\
\hline & 1 NuOver_zo & 3240 & 49400 \\
\hline
\end{tabular}

Table 3. DULBPM entries for leaf texture.

\begin{tabular}{|c|c|c|c|}
\hline \multirow[t]{2}{*}{1} & \multirow{2}{*}{ Leaf_1 } & 6751 & 2745 \\
\hline & & 5109 & 49911 \\
\hline \multirow[t]{2}{*}{2} & \multirow{2}{*}{ Leaf_2 } & 6392 & 3390 \\
\hline & & 5412 & 49322 \\
\hline \multirow[t]{2}{*}{3} & \multirow{2}{*}{ Leaf_3 } & 6613 & 2628 \\
\hline & & 4566 & 50709 \\
\hline \multirow[t]{2}{*}{4} & \multirow{2}{*}{ Leaf_4 } & 8659 & 4601 \\
\hline & & 3016 & 48240 \\
\hline \multirow[t]{2}{*}{5} & \multirow{2}{*}{ Leaf_5 } & 9493 & 2363 \\
\hline & & 2786 & 49874 \\
\hline \multirow[t]{2}{*}{6} & \multirow{2}{*}{ Leaf_6 } & 8983 & 2036 \\
\hline & & 3062 & 50435 \\
\hline \multirow[t]{2}{*}{7} & \multirow{2}{*}{ Leaf_7 } & 5595 & 3166 \\
\hline & & 5072 & 50683 \\
\hline \multirow[t]{2}{*}{8} & \multirow{2}{*}{ Leaf_8 } & 10516 & 2307 \\
\hline & & 2157 & 49536 \\
\hline \multirow[t]{2}{*}{9} & \multirow{2}{*}{ Leaf_9 } & 8751 & 2943 \\
\hline & & 5514 & 47308 \\
\hline \multirow[t]{2}{*}{10} & \multirow{2}{*}{ Leaf_10 } & 9042 & 1179 \\
\hline & & 2313 & 51982 \\
\hline \multirow[t]{2}{*}{11} & \multirow{2}{*}{ Leaf_11 } & 6456 & 2349 \\
\hline & & 3878 & 51833 \\
\hline \multirow[t]{2}{*}{12} & \multirow{2}{*}{ Leaf_12 } & 9353 & 2812 \\
\hline & & 4672 & 47679 \\
\hline \multirow[t]{2}{*}{13} & \multirow{2}{*}{ Leaf_13 } & 7066 & 2556 \\
\hline & & 4213 & 50681 \\
\hline \multirow[t]{2}{*}{14} & & 6177 & 3484 \\
\hline & Leaf_14 & 4378 & 50477 \\
\hline 15 & & 7496 & 2253 \\
\hline & Leat_15 & 3730 & 51037 \\
\hline 16 & & 4885 & 3662 \\
\hline & Leaf_16 & 5155 & 50814 \\
\hline 17 & & 8649 & 1600 \\
\hline & Leaf_17 & 2752 & 51515 \\
\hline 18 & & 31167 & 1328 \\
\hline & Leaf_18 & 1802 & 30219 \\
\hline 19 & & 5191 & 2457 \\
\hline & Leaf_19 & 4055 & 52813 \\
\hline 20 & & 6810 & 2075 \\
\hline & Leaf_20 & 4366 & 51265 \\
\hline
\end{tabular}


Table 4. DULBPM entries for car texture.

\begin{tabular}{|c|c|c|c|}
\hline \multirow[t]{2}{*}{1} & \multirow{2}{*}{ Car_1 } & 30011 & 19 \\
\hline & & 487 & 33999 \\
\hline \multirow[t]{2}{*}{2} & \multirow{2}{*}{ Car_2 } & 18996 & 23 \\
\hline & & 425 & 45072 \\
\hline \multirow[t]{2}{*}{3} & \multirow{2}{*}{ Car_3 } & 28303 & 673 \\
\hline & & 1863 & 33677 \\
\hline \multirow[t]{2}{*}{4} & \multirow{2}{*}{ Car_4 } & 27217 & 9 \\
\hline & & 435 & 36855 \\
\hline \multirow[t]{2}{*}{5} & \multirow{2}{*}{ Car_5 } & 31025 & 10 \\
\hline & & 363 & 33118 \\
\hline \multirow[t]{2}{*}{6} & \multirow{2}{*}{ Car_6 } & 29912 & 7 \\
\hline & & 657 & 33940 \\
\hline \multirow[t]{2}{*}{7} & \multirow{2}{*}{ Car_7 } & 21218 & 32 \\
\hline & & 481 & 42785 \\
\hline \multirow[t]{2}{*}{8} & \multirow{2}{*}{ Car_8 } & 22528 & 20 \\
\hline & & 375 & 41593 \\
\hline \multirow[t]{2}{*}{9} & \multirow{2}{*}{ Car_9 } & 26010 & 11 \\
\hline & & 444 & 38051 \\
\hline \multirow[t]{2}{*}{10} & \multirow{2}{*}{ Car_10 } & 29910 & 10 \\
\hline & & 389 & 34207 \\
\hline \multirow[t]{2}{*}{11} & \multirow{2}{*}{ Car_11 } & 22297 & 15 \\
\hline & & 248 & 41956 \\
\hline \multirow[t]{2}{*}{12} & \multirow{2}{*}{ Car_12 } & 22710 & 10 \\
\hline & & 273 & 41523 \\
\hline \multirow[t]{2}{*}{13} & \multirow{2}{*}{ Car_13 } & 27429 & 26 \\
\hline & & 345 & 36716 \\
\hline \multirow[t]{2}{*}{14} & & 20774 & 12 \\
\hline & Cal_t & 450 & 43280 \\
\hline 15 & & 24073 & 24 \\
\hline & Cai_-10 & 534 & 39885 \\
\hline 16 & & 17184 & 1817 \\
\hline & Cal_tu & 3680 & 41835 \\
\hline 17 & & 12204 & 793 \\
\hline & Cal_li & 3121 & 48398 \\
\hline 18 & & 13406 & 1024 \\
\hline & Cal_to & 2651 & 47435 \\
\hline 19 & & 18886 & 1699 \\
\hline & Cal_ly & 2289 & 41642 \\
\hline 20 & & 22247 & 7 \\
\hline & Cal_zo & 431 & 41831 \\
\hline
\end{tabular}

Table 5. Retrieval rates of different transitions based on DULBPM entries.

\begin{tabular}{|c|c|c|c|c|}
\hline $\begin{array}{c}\text { ujhTexture } \\
\text { Databases }\end{array}$ & $\begin{array}{c}\text { Retrieval } \\
\text { rates } \\
0-0 \\
\text { Transition } \\
\text { of } \\
\text { DULBPM }\end{array}$ & $\begin{array}{c}\text { Retrieval } \\
\text { rates } \\
0-2 \\
\text { Transition } \\
\text { of } \\
\text { DULBPM }\end{array}$ & $\begin{array}{c}\text { Retrieval } \\
\text { rates } \\
2-0 \\
\text { transition } \\
\text { of } \\
\text { DULBPM }\end{array}$ & $\begin{array}{c}\text { Retrieval } \\
\text { rates } \\
2-2 \\
\text { transition } \\
\text { of } \\
\text { DULBPM }\end{array}$ \\
\hline Animal fur & 33 & 60 & 60 & 33.33 \\
\hline Rubber & 40 & 66.66 & 53.33 & 33.33 \\
\hline Leaf & 46.66 & 66.66 & 66.6 & 40 \\
\hline Car & 73.33 & 86.6 & 93.3 & 46.6 \\
\hline $\begin{array}{c}\text { Average } \\
\text { retrieval } \\
\text { rate }\end{array}$ & 53.3 & 69.98 & 68.3 & 38.315 \\
\hline
\end{tabular}

From the Tables 1, 2, 3 and 4 it is clearly evident that the histogram of DULBPM $(2,2)$ shows a very high values for all the considered textures. The reason for this is there are 12 four bit LBP codes that shows exactly two transitions from 0 to 1 or 1 to 0 on each of the corner and diagonal LBP. That why the $\operatorname{DULBPM}(2,2)$ resulted a very high values in the above tables. These histograms of DULBPM $(2,2)$ have no significant ranges of difference among the considered textures because the present paper integrated all 12 LBP four bit codes under one label. The reason for significant improvement for texture retrieval using $\operatorname{DULBPM}(0,2)$ and $\operatorname{DULBPM}(2,0)$ is a relation between 2 codes (with 0 transitions) versus 12 codes (with 2 transitions) of 4 bit corner and diagonal LBP and also vice versa. Ojala et. al [27] proved $80 \%$ to $90 \%$ of textures contains uniform LBP windows on $3 \times 3$ neighborhood i.e only $10 \%$ to $15 \%$ windows are nonuniform LBP. The present paper outlines all $100 \%$ dual LBP's each with 4 bits on a $3 \times 3$ neighborhood are uniform. Therefore there is no rule for NULBP's on the considered DULBPM for any kind of application.

\section{CONCLUSIONS}

The problems related to, which features of image gives best CBIR result remains unsolved. To address this present research focused CBIR based on LBP. LBP characterizes the all significant local image content with higher levels of semantics. One of the disadvantages with LBP is its high dimensionality. The proposed DULBPM reduced the overall dimensionality of evaluating the histograms of 256 patterns of LBP and also 59 patterns of ULBP. The low dimensionality and accuracy of the DULBPM made the LBP to be more suitable to large volumes of data (big-data) and to real time applications. In the proposed DULBPM one has to evaluate only the histograms of four features instead of 256 and 59. Further the present paper also evaluated the transitions of nonuniform LBP's that is $\operatorname{DLBPM}(4,0), \operatorname{DLBPM}(4,2)$, $\operatorname{DLBPM}(4,4), \operatorname{DLBPM}(0,4)$ and they resulted zero only. This clearly indicates that in the considered diagonal LBP the frequency occurrences of 4 or NULBP 
transitions are zero. The retrieval rates of DULBPM $(0,2)$ and DULBPM $(2,0)$ are higher when compared to the other DULBPM entries.

\section{REFERENCES}

[1] Akgül, C.B., Rubin, D.L., Napel, S., Beaulieu, C.F., Greenspan, H., Acar, B., 2011. Content-based image retrieval in radiology: current status and future directions. J. Digital Imag. 24, 208-222.

[2] Aigrain, P., Zhang, H., Petkovic, D., 1996. Content-based representation and retrieval of visual media: a state-of-theart review. Multimedia Tools Appl. 3, 179-202.

[3] Mojsilovic, A., Rogowitz, B., 2001. Capturing image semantics with low-level descriptors. In: Proceedings of the IEEE International Conference on Image Processing, pp. 18-21.

[4] G. Marchionini, Information-seeking strategies of novices using a full-text electronic encyclopedia, J. Amer. Soc. Inform. Sci. 40 (1) (1989) 54-66.

[5] K. Ericsson, A. Lehmann, Expert and exceptional performance: Evidence of maximal adaptation to task constraints, Annu. Rev. Psychol. 47 (1) (1996), 273-305.

[6] T.Q. Lee, Y. Park, Y.T. Park, A time-based approach to effective recommender systems using implicit feedback, Expert Syst. Appl. 34 (4) (2008) 3055- 3062.

[7] S. Krug, Don't Make Me Think!, New Riders Publishing, 2006.

[8] A. Spink, R. Losee, Feedback in information retrieval, Annu. Rev. Inform. Sci. Technol. 31 (1996) 33-78.

[9] I. Lopatovska, I. Arapakis, Theories, methods and current research on emotions in library and information science, information retrieval and human- computer interaction, Inform. Process.Manag. 47 (4) (2011) 575-592.

[10] A. Oulasvirta, J. Hukkinen, B. Schwartz, When more is less: The paradox of choice in search engine use, 2009, pp. 516-523.

[11] B. Poblete, R. Baeza-Yates, Query-sets: Using implicit feedback and query patterns to organize Web documents, 2008, pp. 41-50.

[12] Y. Xu, D. Wang, Order effect in relevance judgment, J. Amer. Soc. Inform. Sci. Technol. 59 (8) (2008) 1264 1275.

[13] Z. Lei, L. Fuzong, Z. Bo, A CBIR method based on colorspatial feature, in: TENCON, Proceedings of the IEEE Region 10 Conference, 1999, pp. 166-169.

[14] H.B. Kekre, S.D. Thepade, Color traits transfer to grayscale images, in: IEEE Int. Conference on Emerging Trends in Engineering and Technology, ICETET, 2008.

[15] C.M. Pun, C.F. Wong, Fast and robust, color feature extraction for content-based image retrieval, Int. J. Adv. Comput. Technol. 3 (6) (2011).

[16] A. Vadivel, S. Sural, A.K. Majumdar, Human color perception in the HSV space and its application in histogram generation for image Retrieval2, in: Proc. SPIE, Color Imaging X: Processing, Hardcopy, and Applications, 2005, pp. 598-609.

[17] C.-R. Shyu, C. E. Brodley, A. C. Kak, A. Kosaka, A. M. Aisen, and L. S. Broderick, "Assert: A physician-in-theloop content-based retrievalsystem for HRCT image databases," Comput. Vis. Image Understand., vol. 75, no. 1-2, pp. 111-132, 1999.

[18] L. Zheng, A. Wetzel, J. Gilbertson, and M. Becich, "Design and analysis of a content-based pathology image retrieval system," IEEE Trans. Inf.Technol. Biomed., vol. 7, no. 4, pp. 249-255, Dec. 2003.
[19] L. Yang, O. Tuzel,W. Chen, P.Meer, G. Salaru, L.Goodell, and D. Foran, "Pathminer: Aweb-based tool for computerassisted diagnostics in pathology," IEEE Trans. Inf. Technol. Biomed., vol. 13, no. 3, pp. 291-299, May,2009.

[20] Liao, M.Law, Chung, "Dominant Local Binary Patterns for Texture Classification”, IEEE Transactions on Image Processing, 2009; 18(5):1107-1118.

[21] G.Zhao, M.Pietik€ainen., "Dynamic texture recognition using local binary patterns with an application to facial expressions", IEEE Transactions on Pattern Analysis and Machine Intelligence (PAMI), 2007; 29 (6):915-928.

[22] W.Zhang, S.Shan, W.Gao, X.Chen, H.Zhang, "Local Gabor binary pattern histogram sequence (LGBPHS): a novel non-statistical model for face representation and recognition", 10th IEEE International Conference on Computer Vision (ICCV), 2005: 786-791.

[23] S.Zhang, H.Yao, SLiu, "Dynamic back ground modeling and subtraction using spatiotemporal local binary patterns", International Conference on image processing (ICIP), 2008: 1556-1559.

[24] Z.Guo, L.Zhang, D.Zhang, "Rotation in variant texture classification using LBP variance (LBPV) with global matching", Pattern Recognition, 2010: 43(3): 706-719.

[25] S.Xie, S.Shan, X.Chen, W.Gao, "V-LGBP: volume based local Gabor binary patterns for face representation and recognition", in: Proceedings of the 19th International Conference on Pattern Recognition (ICPR), 2008:1-4.

[26] S.Liao, X.Zhu, Z.Lei, L.Zhang, S.Z.Li, "Learning multiscale block local binary patterns for face recognition", in: Proceedings of the International Conference on Biometrics (ICB), 2007; 828-837.

[27] Ojala T.; Pietikäinen M.; Mäenpää T.; "Multi resolution Gray-Scale and Rotation Invariant Texture Classification with Local Binary Patterns”, IEEE Trans., 24(7), 2002, pp. 971-987.

[28] Li, Z.; Liu, G.; Yang, Y.; You, J.; "Scale- and rotationinvariant local binary pattern using scale-adaptive texton and sub uniform-based circular shift", IEEE Trans. Image Process, 21(4), 2012, pp. 2130-2140.

[29] Fathi, Abdolhossein, and Ahmad Reza Naghsh-Nilchi. "Noise tolerant local binary pattern operator for efficient texture analysis." Pattern Recognition Letters 33, no. 9 (2012): 1093-1100.

[30] Heikkila, Marko, Matti Pietikainen, and Cordelia Schmid. "Description of interest regions with local binary patterns." Pattern recognition 42, no. 3 (2009): 425-436.

[31] Liao, Shu, Max WK Law, and Albert CS Chung. "Dominant local binary patterns for texture classification." Image Processing, IEEE Transactions on 18, no. 5 (2009): 1107-1118.

[32] Murty, Gorti Satyanarayana, J. Sasi Kiran, and V. Vijaya Kumar. "Facial Expression Recognition Based on Features Derived From the Distinct LBP and GLCM." International Journal of Image, Graphics and Signal Processing (IJIGSP) 6, no. 2 (2014): 68.

[33] Nanni, Loris, Sheryl Brahnam, and Alessandra Lumini. "A simple method for improving local binary patterns by considering non-uniform patterns." Pattern Recognition 45, no. 10 (2012): 3844-3852.

[34] V.Vijaya Kumar, K.Srinivasa Reddy, V.V.Krishna, "Face recognition using prominent LBP model", International Journal of Applied Engineering Research (IJAER), Volume 10, No.2(2015), pp 4373-4384.

[35] Zhou, Hui, Runsheng Wang, and Cheng Wang. "A novel extended local-binary-pattern operator for texture analysis." Information Sciences 178, no. 22 (2008): 43144325. 


\section{Authors' Profiles}

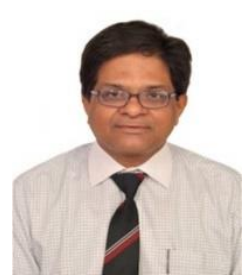

Dr. V. Vijaya Kumar is working as Dean in Dept. of CSE \& IT and Director- Centre for Advanced Computational Research (CACR) at Anurag Group of Institutions, (AGOI) (Autonomous), Hyderabad. He received integrated M.S.Engg, in CSE from USSR in 1989. He received his Ph.D. degree in Computer Science from Jawaharlal Nehru Technological University (JNTU), Hyderabad, India in 1998 and guided 22 research scholars for Ph.D. He has served JNT University for 13 years as Assistant Professor and Associate Professor. He has received best researcher and best teacher award from JNT University, Kakinada, India. His research interests include Image Processing, Pattern Recognition, Digital Water Marking, Cloud Computing, Image Retrieval Systems and image analytics in Big Data. He is the life member of CSI, ISCA, ISTE, IE (I), IETE, ACCS, CRSI, IRS and REDCROSS. He published more than 120 research publications till now in various National, International journals and conferences. He has also established and also acted as a Head, Srinivasa Ramanujan Research Forum (SRRF) at GIET, Rajahmundry, India for promoting research and social activities. as free-lance programmer. He is Organizer, Advisory member for various National, International Conferences in the field of Information Technology. Member in various Professional Bodies like ISPACE, ASCAP, IACSIT etc.

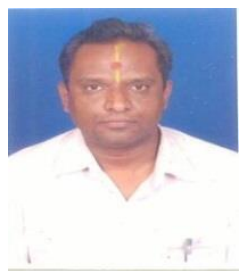

Y. K. Sundara Krishna qualified in Ph.D in Computer Science \& Engineering from Osmania University, Hyderabad. Now, he is working as Professor in the Department of Computer Science, Krishna University, and Machilipatnam. His research interests are Mobile Computing, Service Oriented Architecture, image processing and having practical work experience in the areas of Computing Systems including Developing of Simulators for Distributed Dynamic Cellular Computing Systems, Applications of Embedded \& Win32 clients, Maintenance of Multi-user System Software. Also he is working with International Telecommunications Union (ITU): Y. 2018 recommendation series Y: Global Information Infrastructure, Internet Protocol aspects and NGN.

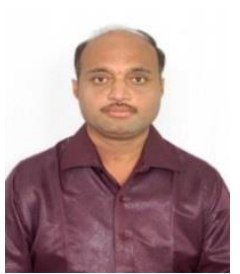

A. Srinivasa Rao $\mathrm{He}$ is presently working as Principal (i/c)\& Assoc. professor, Head; C.Sc.Dept Montessori Siva Sivani institute of Science \& Technology College of Engineering Mylavaram. He is pursuing Ph.d from Krishna University, Machalipatnam under the guidance of Prof.V.Venkata Krishna. He got 19 years of teaching experience and taught various courses to UG and PG programs. He served industry for 6 years

How to cite this paper: V.Vijaya Kumar, A. Srinivasa Rao, YK Sundara Krishna,"Dual Transition Uniform Lbp Matrix for Efficient Image Retrieval", IJIGSP, vol.7, no.8, pp.50-57, 2015.DOI: 10.5815/ijigsp.2015.08.06 